\title{
A Principled Approach to Mixed Integer/Linear Problem Formulation
}

\author{
J. N. Hooker
}

September 9, 2008

\begin{abstract}
We view mixed integer/linear problem formulation as a process of identifying disjunctive and knapsack constraints in a problem and converting them to mixed integer form. We show through a series of examples that following this process can yield mixed integer models that automatically incorporate some of the modeling devices that have been discovered over the years for making the formulation tighter. In one case it substantially improves on the generally accepted model. We provide a theoretical basis for the process by generalizing Jeroslow's mixed integer representability theorem.
\end{abstract}

\section{Introduction}

Mixed integer problem formulation is an art rather than a science, but it need not be unprincipled. A theorem of Jeroslow [4], for example, provides guidance for writing formulations. It states that a problem can be given a mixed integer/linear formulation if and only if its feasible set is a union of finitely many polyhedra that satisfy a certain technical condition.

This suggests a disjunctive approach to mixed integer formulation. A union of polyhedra is represented by a disjunction of linear systems. So if we can understand a problem as presenting choices between discrete alternatives, we can perhaps write the choices as disjunctions of linear systems and convert each disjunction to a mixed integer formulation. In this way we obtain a mixed integer formulation for the entire problem.

Jeroslow's disjunctive formulations have the additional advantage that each disjunction receives a convex hull formulation, the tightest possible mixed integer/linear formulation. The continuous relaxation of the formulation describes the convex hull of the feasible set of the disjunction. 
The disjunctive approach provides a useful device for creating mixed integer formulations for many problems, but in other cases it is impractical due to the large number of disjunctions required. Integer knapsack constraints are particularly troublesome, because the feasible set is a finite union of polyhedra only in the technical sense that each integer point is a polyhedron. Even this assumes that the feasible set is finite, and Jeroslow's theorem is in fact valid only when the integer variables in the mixed integer formulation are bounded. A purely disjunctive approach is therefore impractical and unnatural when the problem contains integer knapsack constraints, as many do.

We therefore propose that mixed integer/linear formulation combines two quite different kinds of ideas: disjunctions and integer knapsack constraints. We suggest that by identifying these two elements in a given problem, one can obtain practical mixed integer formulations in a reasonably principled way. Some of these formulations automatically incorporate nonobvious devices for tightening the formulation that are part of the folklore of modeling. In at least one case, the formulation is even better than the generally accepted one.

We ground this approach theoretically by extending Jeroslow's theorem in a straightforward way. We show that a problem has a mixed integer/linear formulation if and only if its feasible set is a union of finitely many mixed integer polyhedra satisfying a technical condition. A mixed integer polyhedron is, roughly speaking, a polyhedron in which some or all of the variables are required to be integer.

This is more general than Jeroslow's theorem because it allows for unbounded integer variables. It also incorporates integer knapsack constraints in a natural way, because disjunctions of linear systems become disjunctions of inequality systems that may contain integer knapsack inequalities. A problem consisting entirely of integer knapsack inequalities is a special case in which the formulation contains one disjunct. We also show that each disjunction receives a convex hull formulation, provided the individual disjuncts are convex hull formulations.

Williams points out in [8] that a representable union of polyhedra can always be given a "big- $M$ " formulation as well as a convex hull formulation. The big- $M$ formulation is generally not as tight but contains fewer variables. Thus Jeroslow's representability theorem does not rely specifically on giving a convex hull formulation to disjunctions. We show that the same holds for general mixed integer representability. Any representable union of mixed integer polyhedra can be given a big- $M$ mixed integer formulation as well as a convex hull formulation. 
The paper has two main parts. The first deals with purely disjunctive formulations, while the second incorporates integer knapsack constraints. The first part begins with Jeroslow's result and illustrates it with a fixed charge problem. It also discusses the issue of when it is advantageous to combine several disjunctions into one long disjunction. Formulations are then derived for capacitated and uncapacitated facility location problems, using the disjunctive approach. The uncapacitated formulation avoids a typical beginner's mistake and thus shows how one may sidestep such pitfalls by following a principled method. Next, a lot sizing problem illustrates how logical constraints can assist problem formulation, although they can in principle be eliminated. Finally, we discuss big- $M$ disjunctive formulations.

The second main part of the paper begins by generalizing Jeroslow's representability theorem, using both convex hull and big- $M$ disjunctive formulations. We then formulate a modified facility location problem in which discrete variables account for the number of vehicles used to transport goods. This example shows how disjunctions of mixed integer systems, rather than linear systems, can occur in problem formulations. A package delivery problem then illustrates how a standard modeling trick falls automatically out of a principled approach. One can therefore obtain a tight model without knowing the "folklore" of modeling. It also illustrates how a principled approach leads one to include a redundant constraint that, according to conventional wisdom, can serve no purpose in the formulation. Nonetheless, this constraint makes the problem much easier to solve.

Some of the disjunctive formulations presented here appear in [3]. Several examples of mixed integer modeling in general can be found in [7].

\section{Disjunctive Formulations}

Disjunctive formulations are useful when one must make a choice from two or more alternatives. Problems typically present several such choices, and a disjunctive constraint can be written for each. If each constraint is a disjunction of linear systems, then it can be given a tight mixed/integer linear formulation, yielding a formulation for the problem as a whole.

We present in this section some examples in which a disjunctive analysis is the natural one. Further examples can be found in [3]. We begin with Jeroslow's result, which provides the theoretical basis for disjunctive formulation. 


\subsection{Bounded Mixed Integer Representability}

Jeroslow $[4,5]$ defined a subset of $\mathbb{R}^{n}$ to be bounded mixed integer representable when it is the feasible set of a linear formulation with continuous and 0-1 variables. More precisely, $S \in \mathbb{R}^{n}$ is representable if there is a constraint set of the following form whose projection onto $x$ is $S$ :

$$
\begin{aligned}
& A x+B u+D y \geq b \\
& x \in \mathbb{R}^{n}, \quad u \in \mathbb{R}^{m}, \quad y_{k} \in\{0,1\}, \text { all } k
\end{aligned}
$$

The continuous variables $u$ and discrete variables $y$ can be viewed as auxiliary variables that help to define the feasible subset of $\mathbb{R}^{n}$.

The discrete variables are restricted to be $0-1$ in this definition, but an equivalent definition can be obtained by replacing the $0-1$ variables with general integer variables - provided the general integer variables are bounded. This is because a bounded integer variable $y_{k}$ can be replaced by $\sum_{j=0}^{p} 2^{j} y_{k j}$, where each $y_{k j}$ is $0-1$, and a system of the form (1) results. Thus the term "bounded" in "bounded mixed integer representability" does not mean that the set to be represented is bounded. It means that the integer variables are bounded.

Jeroslow proved that $S \in \mathbb{R}^{n}$ is representable in this sense if and only if $S$ is a union of finitely many polyhedra that have the same recession cone. The recession cone of a polyhedron $P$ is the set of directions in which $P$ is unbounded, or more precisely, the set of vectors $r \in \mathbb{R}^{n}$ such that, given any $u \in P, u+\beta r \in P$ for all $\beta \geq 0$.

The proof is based on the fact that representability in Jeroslow's sense is equivalent to representability by a disjunctive constraint of the form

$$
\bigvee_{k \in K}\left(A^{k} x \geq b^{k}\right)
$$

where $K$ is finite. The disjunction (2) requires that $x$ satisfy at least one of the linear systems $A^{k} x \geq b^{k}$. Each system $A^{k} x \geq b^{k}$ can be viewed as defining one of the polyhedra $P_{k}$ that make up $S$, so that $S=\bigcup_{k \in K} P_{k}$.

Theorem 1 (Jeroslow) $A$ set $S \subset \mathbb{R}^{n}$ is bounded mixed integer representable if and only if $S$ is the union of finitely many polyhedra having the same recession cone. In particular, $S$ is bounded mixed integer representable if and only if $S$ is the projection onto $x$ of a mixed integer formulation with 
following form:

$$
\begin{aligned}
& x=\sum_{k \in K} x^{k} \\
& A^{k} x^{k} \geq b^{k} y_{k}, \quad k \in K \\
& \sum_{k \in K} y_{k}=1, \quad y_{k} \in\{0,1\}, k \in K
\end{aligned}
$$

The mixed integer formulation (3) represents the disjunctive problem (2). In particular, $y_{k}=1$ when $x$ satisfies the $k$ th disjunct of (2). Note that $x$ is disaggregated into a sum of continuous variables $x^{k}$, which play the role of auxiliary variables $u$ in (1). Thus (3) has the form (1).

The mixed integer formulation (3) not only represents (2) but is a convex hull formulation of (2). That is, the continuous relaxation of (3) has a feasible set that, when projected onto $x$, is the convex hull of the feasible set of (2). The continuous relaxation of (3) is obtained by replacing $y_{k} \in\{0,1\}$ with $y_{k} \geq 0$ for each $k$.

\subsection{Example: Fixed-Charge Function}

Bounded mixed integer representability is illustrated by the fixed-charge function, which occurs frequently in modeling. Suppose the cost $x_{2}$ of manufacturing quantity $x_{1}$ of some product is to be minimized. The cost is zero when $x_{1}=0$ and is $f+c x_{1}$ otherwise, where $f$ is the fixed cost and $c$ the unit variable cost.

The problem can be viewed as minimizing $x_{2}$ subject to $\left(x_{1}, x_{2}\right) \in S$, where $S$ is the set depicted in Figure 1(a). $S$ is the union of two polyhedra $P_{1}$ and $P_{2}$, and the problem is to minimize $x_{2}$ subject to the disjunction

$$
\left(\begin{array}{l}
x_{1}=0 \\
x_{2} \geq 0
\end{array}\right) \vee\left(\begin{array}{c}
x_{2} \geq c x_{1}+f \\
x_{1} \geq 0
\end{array}\right)
$$

where the disjuncts correspond respectively to $P_{1}$ and $P_{2}$. In general there would be additional constraints in the problem, but we focus here on the fixed-charge formulation.

The recession cone of $P_{1}$ is $P_{1}$ itself, and the recession cone of $P_{2}$ is the set of all vectors $\left(x_{1}, x_{2}\right)$ with $x_{2} \geq c x_{1} \geq 0$. Thus, by Theorem $1, S$ is not bounded mixed integer representable. Indeed, the formulation (3) becomes

$$
\begin{array}{llll}
x_{1}=x_{1}^{1}+x_{1}^{2} & x_{1}^{1} \leq 0 & -c x_{1}^{2}+x_{2}^{2} \geq f y_{2} & y_{1}+y_{2}=1 \\
x_{2}=x_{2}^{1}+x_{2}^{2} & x_{1}^{1}, x_{2}^{1} \geq 0 & x_{1}^{2} \geq 0 & y_{1}, y_{2} \in\{0,1\}
\end{array}
$$




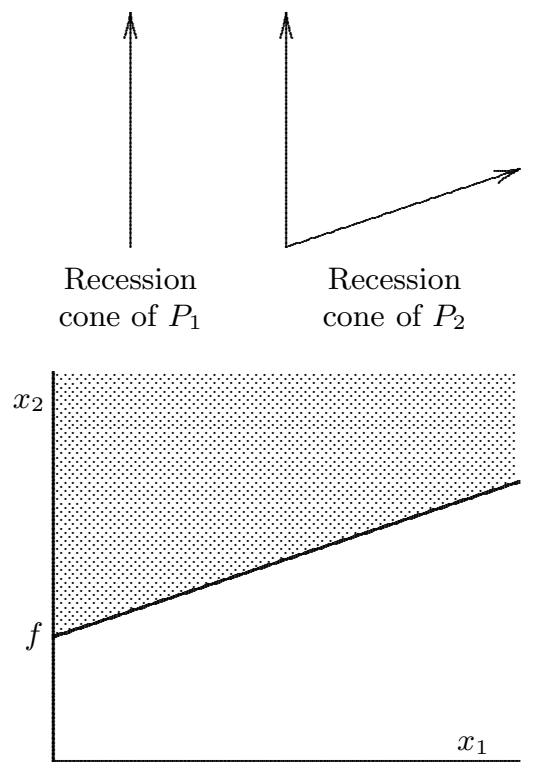

(a)

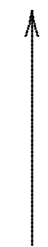

Recession cone of $P_{1}, P_{2}$

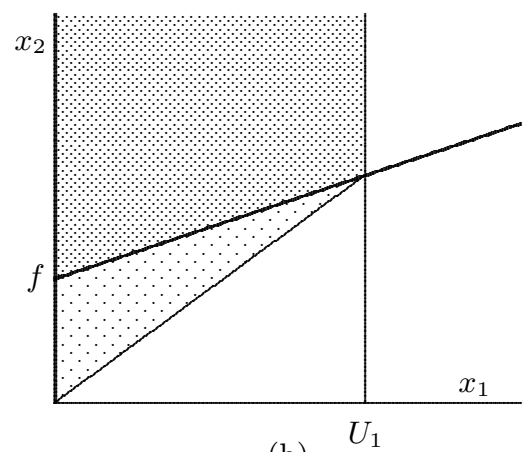

(b)

Figure 1: (a) Feasible set of a fixed-charge problem, consisting of the union of polyhedra $P_{1}$ (heavy vertical line) and $P_{2}$ (shaded area). (b) Feasible set of the same problem with the bound $x_{1} \leq U_{1}$, where $P_{2}^{\prime}$ is the darker shaded area. The convex hull of the feasible set is the entire shaded area.

and does not correctly represent $S$, as can be seen by simplifying (4). Only one 0 - 1 variable appears, which can be renamed $y$. Also, we can set $x_{1}^{2}=x_{1}$ ( since $x_{1}^{1}=0$ ) and $x_{2}^{1}=x_{2}-x_{2}^{2}$, which yields

$$
x_{1} \geq 0, \quad x_{2}-x_{2}^{2} \geq 0, \quad x_{2}^{2}-c x_{1} \geq f y, \quad y \in\{0,1\}
$$

Minimizing $x_{2}$ subject to this is equivalent to minimizing $x_{2}$ subject to

$$
x_{1} \geq 0, \quad x_{2}-c x_{1} \geq f y, \quad y \in\{0,1\}
$$

The projection onto $\left(x_{1}, x_{2}\right)$ is the union of the two polyhedra obtained by setting $y=0$ and $y=1$. The projection is therefore the set of all points satisfying $x_{2} \geq c x_{1}, x_{1} \geq 0$, which is clearly different from $P_{1} \cup P_{2}$. The formulation is therefore incorrect.

However, if we place an upper bound $U_{1}$ on $x_{1}$, the problem is now to 
minimize $x_{2}$ subject to

$$
\left(\begin{array}{l}
x_{1}=0 \\
x_{2} \geq 0
\end{array}\right) \vee\left(\begin{array}{c}
x_{2} \geq c x_{1}+f \\
0 \leq x_{1} \leq U_{1}
\end{array}\right)
$$

The recession cone of each of the resulting polyhedra $P_{1}, P_{2}^{\prime}$ (Figure $1 \mathrm{~b}$ ) is the same (namely, $P_{1}$ ), and the feasible set $S^{\prime}=P_{1} \cup P_{2}^{\prime}$ is therefore bounded mixed integer representable. The convex hull formulation is

$$
\begin{array}{llll}
x_{1}^{1} \leq 0 & -c x_{1}^{2}+x_{2}^{2} \geq f y_{2} & x_{1}=x_{1}^{1}+x_{1}^{2} & y_{1}+y_{2}=1 \\
x_{1}^{1}, x_{2}^{2} \geq 0 & 0 \leq x_{1}^{2} \leq U_{1} y_{2} & x_{2}=x_{2}^{1}+x_{2}^{2} & y_{1}, y_{2} \in\{0,1\}
\end{array}
$$

Again the model simplifies:

$$
x_{1} \leq U_{1} y, \quad x_{2} \geq f y+c x_{1}, \quad x_{1} \geq 0, y \in\{0,1\}
$$

Obviously, $y$ encodes whether the quantity produced is zero or positive, in the former case $(y=0)$ forcing $x_{1}=0$, and in the latter case incurring the fixed charge $f$. Big- $M$ constraints like $x_{1} \leq U_{1} y$, which are very common in mixed integer models, can often be viewed as originating from upper bounds that are imposed to ensure that the polyhedra concerned have the same recession cone.

Big- $M$ s do not always have this origin, however. For example, a disjunctive constraint (2) can be given a big- $M$ disjunctive formulation, which contains fewer continuous variables than a convex hull formulation but may not be as tight. This type of formulation is discussed further in Section 2.6.

\subsection{Multiple Disjunctions}

A mixed integer formulation may consist of multiple convex hull formulations, one for each disjunction. Such a formulation does not in general provide a convex hull relaxation for the problem as a whole. Consider, for example, the constraint set

$$
\begin{aligned}
& \left(\begin{array}{c}
x_{1}=0 \\
x_{2} \in[0,1]
\end{array}\right) \vee\left(\begin{array}{c}
x_{2}=0 \\
x_{1} \in[0,1]
\end{array}\right) \\
& \left(\begin{array}{c}
x_{1}=0 \\
x_{2} \in[0,1]
\end{array}\right) \vee\left(\begin{array}{c}
x_{2}=1 \\
x_{1} \in[0,1]
\end{array}\right)
\end{aligned}
$$

The convex hull formulations of the two disjunctions are

$$
\begin{aligned}
& 0 \leq x_{1} \leq 1-y_{1}, \quad 0 \leq x_{2} \leq y_{1}, \quad y_{1} \in\{0,1\} \\
& 0 \leq x_{1} \leq 1-y_{2}, \quad 1-y_{2} \leq x_{2} \leq 1, \quad y_{2} \in\{0,1\}
\end{aligned}
$$




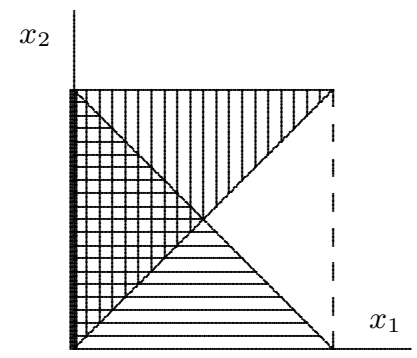

Figure 2: Convex hull relaxation of (8a) (horizontal shading), convex hull relaxation of (8b) (vertical shading), continuous relaxation of (8) (heavy shading), and convex hull relaxation of (7) (heavy vertical line segment).

The feasible set of (8), projected onto $x_{1}, x_{2}$, is the heavy vertical line segment in Fig. 2, and its convex hull is the same line segment. The convex hulls described by continuous relaxations of (a) and (b) are

$$
\begin{aligned}
& x_{1}+x_{2} \leq 1, \quad x_{1}, x_{2} \geq 0 \\
& x_{1} \leq x_{2}, \quad x_{1} \geq 0, \quad x_{2} \leq 1
\end{aligned}
$$

and also appear in the figure. The continuous relaxation of (8) corresponds to the intersection of these two convex hulls and is therefore weaker than a convex hull relaxation of $(8)$.

A convex hull formulation can always be obtained for multiple disjunctions by taking the product of the disjunctions to obtain a single disjunction, which can then be given a convex hull formulation. That is, two disjunctions $A \vee B$ and $C \vee D$ can be written as a product $A C \vee A D \vee B C \vee B D$, where $A C$ refers to the linear system consisting of both $A$ and $C$. For example, the two disjunctions of (7) yield the product

$$
\left(\begin{array}{c}
x_{1}=0 \\
x_{2} \in[0,1]
\end{array}\right) \vee\left(\begin{array}{c}
x_{1}=0 \\
x_{2}=1 \\
x_{1}, x_{2} \in[0,1]
\end{array}\right) \vee\left(\begin{array}{c}
x_{1}=0 \\
x_{2}=0 \\
x_{1}, x_{2} \in[0,1]
\end{array}\right) \vee\left(\begin{array}{c}
x_{2}=0 \\
x_{2}=1 \\
x_{1} \in[0,1]
\end{array}\right)
$$

The mixed integer formulation of (10) simplifies to $x_{1}=0,0 \leq x_{2} \leq 1$.

Although the convex hull formulation of the product simplifies in this example, formulating a product of disjunctions is not in general a practical option because the number of disjuncts grows exponentially. However, it may be useful to take a product of certain subsets of disjunctions. This can strengthen the relaxation, but only when the disjunctions have variables in common, due to the following lemma. 
Lemma 2 If two disjunctions $D_{1}, D_{2}$ of linear systems have no variables in common, then the convex hull formulations of $D_{1}$ and $D_{2}$, when taken together, already provide a convex hull formulation of $\left\{D_{1}, D_{2}\right\}$.

Proof. Let $F_{i}$ be the feasible set of $D_{i}$, for $i=1,2$. It suffices to show that $\operatorname{conv}\left(F_{1} \cap F_{2}\right)=\operatorname{conv}\left(F_{1}\right) \cap \operatorname{conv}\left(F_{2}\right)$, where $\operatorname{conv}\left(F_{i}\right)$ is the convex hull of $F_{i}$. Obviously, $\operatorname{conv}\left(F_{1} \cap F_{2}\right) \subset \operatorname{conv}\left(F_{1}\right) \cap \operatorname{conv}\left(F_{2}\right)$. To show that $\operatorname{conv}\left(F_{1}\right) \cap \operatorname{conv}\left(F_{2}\right) \subset \operatorname{conv}\left(F_{1} \cap F_{2}\right)$, take any $\bar{x} \in \operatorname{conv}\left(F_{1}\right) \cap \operatorname{conv}\left(F_{2}\right)$, and let $\bar{x}=(\bar{u}, \bar{v})$, where $u$ and $v$ consist of the variables in $D_{1}$ and $D_{2}$, respectively. Since $\bar{x} \in \operatorname{conv}\left(F_{1}\right)$,

$$
(\bar{u}, \bar{v})=\alpha\left(a^{1}, c^{2}\right)+(1-\alpha)\left(b^{1}, d^{2}\right)
$$

where $\alpha \in[0,1]$ and $\left(a^{1}, c^{2}\right),\left(b^{1}, d^{2}\right) \in F_{1}$. Similarly,

$$
(\bar{u}, \bar{v})=\beta\left(c^{1}, a^{2}\right)+(1-\beta)\left(d^{1}, b^{2}\right)
$$

where $\beta \in[0,1]$ and $\left(c^{1}, a^{2}\right),\left(d^{1}, b^{2}\right) \in F_{2}$. Using (11)-(12), it can be readily checked that $(\bar{u}, \bar{v})$ is a convex combination of four points:

$$
\alpha \beta\left(a^{1}, a^{2}\right)+\alpha(1-\beta)\left(a^{1}, b^{2}\right)+(1-\alpha) \beta\left(b^{1}, a^{2}\right)+(1-\alpha)(1-\beta)\left(b^{1}, b^{2}\right)
$$

where (11) is used to verify the first component $\bar{u}$ and (12) to verify the second component $\bar{v}$. But $\left(a^{1}, a^{2}\right) \in F_{1} \cap F_{2}$ because $\left(a^{1}, c^{2}\right) \in F_{1},\left(c^{1}, a^{2}\right) \in$ $F_{2}$, and $D_{1}$ and $D_{2}$ have no variables in common. Similarly, the other three points belong to $F_{1} \cap F_{2}$, and $\bar{x} \in \operatorname{conv}\left(F_{1} \cap F_{2}\right)$.

\subsection{Example: Facility Location}

A simple capacitated facility location problem illustrates how a disjunctive formulation can be developed in practice. There are $m$ possible locations for facilities, and $n$ customers who obtain products from the facilities. A facility installed at location $i$ incurs fixed cost $f_{i}$ and has capacity $C_{i}$. Each customer $j$ has demand $D_{j}$, and the unit cost of shipping from facility $i$ to customer $j$ is $c_{i j}$. The problem is to decide which facilities to install, and how to supply the customers, so as to minimize total fixed and variable costs.

Each location $i$ either receives a facility or not. If it does, the total shipments out of the location must be at most $C_{i}$, and a fixed cost is incurred. Otherwise nothing is shipped out of the location. Thus if $x_{i j}$ is the quantity 
shipped from $i$ to $j$, we have the disjunction

$$
\left(\begin{array}{c}
\sum_{j=1}^{n} x_{i j} \leq C_{i} \\
x_{i j} \geq 0, \text { all } j \\
z_{i}=f_{i}
\end{array}\right) \vee\left(\begin{array}{c}
x_{i j}=0, \text { all } j \\
z_{i}=0
\end{array}\right)
$$

where $z_{i}$ represents the fixed cost incurred at location $i$. In addition, each customer $j$ must receive adequate supply:

$$
\sum_{i=1}^{m} x_{i j}=D_{j}, \text { all } j
$$

This can be viewed as a disjunction with one disjunct. The problem is to minimize

$$
\sum_{i=1}^{m}\left(z_{i}+\sum_{j=1}^{n} c_{i j} x_{i j}\right)
$$

subject to (14) and (15).

Rather than writing a convex hull formulation for the product of the disjunctions (14) and the disjunction (15), which is a very complicated matter, we can formulate each disjunction individually. The convex hull formulation of $(14)$ is

$$
\sum_{j=1}^{n} x_{i j} \leq C_{i} y_{i}, \quad z_{i}=f_{i} y_{i}, \quad y_{i} \in\{0,1\}, \quad x_{i j} \geq 0, \text { all } j
$$

and (15) is its own convex hull formulation. A mixed integer formulation can now be obtained by minimizing (16) subject to (15) and (17) for all $i$. This immediately simplifies to

$$
\begin{aligned}
& \min \sum_{i=1}^{m}\left(f_{i} y_{i}+\sum_{j=1}^{n} c_{i j} x_{i j}\right) \\
& \sum_{j=1}^{n} x_{i j} \leq C_{i} y_{i}, \text { all } i \\
& \sum_{i=1}^{m} x_{i j}=D_{j}, \text { all } j \\
& y_{i} \in\{0,1\}, x_{i j} \geq 0, \text { all } i, j
\end{aligned}
$$


This formulation is succinct enough, and its continuous relaxation tight enough, to be useful in practice.

A disjunctive approach to formulation can sometimes lead to tighter relaxations than one would obtain otherwise. A common beginner's mistake, for example, is to model the uncapacitated facility location problem as a special case of the capacitated problem. In the uncapacitated problem, there is no limit on the capacity of each facility, and $x_{i j}$ represents the fraction of customer $j$ 's demand supplied by facility $i$, so that each $D_{j}=1$. Although there is no capacity limit, one can observe that each facility will ship at most $n$ units and therefore let $C_{i}=n$ in the formulation (18) for the capacitated problem. This is a valid formulation of the uncapacitated problem, but there is a much tighter one.

We start with a disjunctive conception of the problem. If facility $i$ is installed, it supplies at most one unit to each customer and incurs cost $f_{i}$. If it is not installed, then it supplies nothing:

$$
\left(\begin{array}{c}
0 \leq x_{i j} \leq 1, \text { all } j \\
z_{i}=f_{i}
\end{array}\right) \vee\left(\begin{array}{c}
x_{i j}=0, \text { all } j \\
z_{i}=0
\end{array}\right)
$$

The convex hull formulation of this disjunction is

$$
z_{i}=f_{i} y_{i}, y_{i} \in\{0,1\}, 0 \leq x_{i j} \leq y_{i}, \text { all } j
$$

This yields a tighter formulation than (18):

$$
\begin{aligned}
& \min \sum_{i=1}^{m}\left(f_{i} y_{i}+\sum_{j=1}^{n} c_{i j} x_{i j}\right) \\
& x_{i j} \leq y_{i}, \text { all } i, j \\
& \sum_{i=1}^{m} x_{i j}=1, \text { all } j \\
& y_{i} \in\{0,1\}, \quad x_{i j} \geq 0, \text { all } i, j
\end{aligned}
$$

To see that it is tighter, note first that constraints in (18) and (20) are the same except for (b), and that (20b) implies (18b) because the latter is the sum of the constraints in the former. Furthermore, setting (for example) $y_{i}=1 / 2$ for each $i, x_{i j}=0$ for each $i$ and $j \leq n / 2$, and $x_{i j}=1$ for each $i$ and $j>n / 2$ (supposing $n$ is even) satisfies the continuous relaxation of (18) but not that of $(20)$.

This is an instance in which the more succinct relaxation is not the tighter one. The smaller formulation (18) with only $2 m$ constraints (other than variable bounds) is not as tight as $(20)$, which has $m(n+1)$ constraints. 


\subsection{Example: Lot Sizing with Setup Costs}

A lot sizing problem with set up costs illustrates how logical relations among linear systems can be captured with logical constraints that involve the $0-1$ variables. Logical constraints do not enhance the representability of mixed integer formulations, but they may be convenient in practice.

In the lot sizing problem, here is a demand $D_{t}$ for a product in each period $t$. No more than $C_{t}$ units of the product can be manufactured in period $t$, and any excess over demand is stocked to satisfy future demand. If there is no production in the previous period, then a setup cost of $f_{t}$ is incurred. The unit production cost is $p_{t}$, and the unit holding cost per period is $h_{t}$. A starting stock level $s_{0}$ is given. The objective is to choose production levels in each period so as to minimize total cost over all periods.

Let $x_{t}$ be the production level in period $t$ and $s_{t}$ the stock level at the end of the period. In each period $t$, there are three options to choose from: (1) start producing (with a setup cost), (2) continue producing (with no setup cost), and (3) produce nothing. If $v_{t}$ is the setup cost incurred in period $t$, these correspond respectively to the three disjuncts

$$
\left(\begin{array}{c}
v_{t} \geq f_{t} \\
0 \leq x_{t} \leq C_{t}
\end{array}\right) \vee\left(\begin{array}{c}
v_{t} \geq 0 \\
0 \leq x_{t} \leq C_{t}
\end{array}\right) \vee\left(\begin{array}{c}
v_{t} \geq 0 \\
x_{t}=0
\end{array}\right)
$$

There are logical connections between the choices in consecutive periods. If we schematically represent the disjunction (21) as

$$
Y_{t} \vee Z_{t} \vee W_{t}
$$

the logical connections can be written

$$
\begin{aligned}
& Z_{t} \Rightarrow\left(Y_{t-1} \vee Z_{t-1}\right) \\
& Y_{t} \Rightarrow\left(\neg Y_{t-1} \wedge \neg Z_{t-1}\right)
\end{aligned}
$$

where $\neg$ means "not" and $\wedge$ means "and." The inventory balance constraints are

$$
s_{t-1}+x_{t}=D_{t}+s_{t}, \quad s_{t} \geq 0, \quad t=1, \ldots, n
$$

where $s_{t}$ is the stock level in period $t$ and $s_{0}$ is given. The problem is to minimize

$$
\sum_{t=1}^{n}\left(p_{t} x_{t}+h_{t} s_{t}+v_{t}\right)
$$

subject to (21) and (23) for all $t \geq 1$ and (24). 
A convex hull formulation for $(21)$ is

$$
\begin{array}{lll}
v_{t}^{1} \geq f_{t} y_{t}, & v_{t}^{2} \geq 0, & v_{t}^{3} \geq 0 \\
0 \leq x_{t}^{1} \leq C_{t} y_{t}, \quad 0 \leq x_{t}^{2} \leq C_{t} z_{t}, & x_{t}^{3}=0 \\
v_{t}=v_{t}^{1}+v_{t}^{2}+v_{t}^{3} & x_{t}=x_{t}^{1}+x_{t}^{2}+x_{t}^{3} & \\
y_{t}+z_{t}+w_{t}=1, & y_{t}, z_{t}, w_{t} \in\{0,1\} &
\end{array}
$$

Thus, $z_{t}=1$ indicates a startup, $y_{t}=1$ continued production, and $w_{t}=1$ no production in period $t$. To simplify $(26)$, we first eliminate $w_{t}$, so that $y_{t}+z_{t} \leq 1$. Since $x_{t}^{3}=0$, we can set $x_{1}=x_{1}^{1}+x_{2}^{2}$, which allows us to replace the two capacity constraints in (26) by $0 \leq x_{t} \leq C_{t}\left(y_{t}+z_{t}\right)$. Finally, $v_{t}$ can replace $v_{t}^{1}$, because $v_{t}$ is being minimized and $v_{t}^{2}$ and $v_{t}^{3}$ do not appear. The convex hull formulation (26) becomes

$$
\begin{aligned}
& v_{t} \geq f_{t} y_{t}, \quad 0 \leq x_{t} \leq C_{t}\left(y_{t}+z_{t}\right) \\
& y_{t}+z_{t} \leq 1, \quad y_{t}, z_{t} \in\{0,1\}
\end{aligned}
$$

The logical constraints (23) can be formulated

$$
z_{t} \leq y_{t-1}+z_{t-1}, \quad y_{t} \leq 1-y_{t-1}-z_{t-1}
$$

The second constraint is correct because we know $y_{t}+z_{t} \leq 1$. The entire problem can now be formulated as minimizing (25) subject to (24) and (27)-(28) for all $t \geq 1$.

The problem can also be formulated without logical constraints. We first write the logical constraints (23) as a set of disjunctions (i.e., in conjunctive normal form):

$$
\begin{aligned}
& \neg Z_{t} \vee Y_{t-1} \vee Z_{t-1} \\
& \neg Y_{t} \vee \neg Y_{t-1} \\
& \neg Y_{t} \vee \neg Z_{t-1}
\end{aligned}
$$

We now replace each negated term with the disjunction of the remaining terms in the disjunction (22) that contains it:

$$
\begin{aligned}
& Y_{t} \vee W_{t} \vee Y_{t-1} \vee Z_{t-1} \\
& Z_{t} \vee W_{t} \vee Z_{t-1} \vee W_{t-1} \\
& Z_{t} \vee W_{t} \vee Y_{t-1} \vee W_{t-1}
\end{aligned}
$$

We can now drop the logical constraints (28) and add convex hull formulations of the disjunctions in (29). This kind of maneuver can sometimes result in a tighter formulation, but it may not be worth the additional variables and constraints. 


\subsection{Big- $M$ Disjunctive Formulations}

A disjunction (2) of linear systems can be given a big- $M$ disjunctive formulation as well as a convex hull formulation. The big- $M$ formulation has fewer variables because the continuous variables are not disaggregated. It may be preferable in practice when there are a large number of disjuncts, even though its continuous relaxation can be significantly weaker than that of a convex hull formulation.

As noted earlier, Jeroslow's bounded representability theorem does not rely specifically on a convex hull formulation of disjunctions [8]. Any finite union of polyhedra with the same recession cone can be given a big- $M$ formulation as well as a convex hull formulation. We extend this result to general representability in Section 3.2.

A big- $M$ disjunctive formulation for (2) has the form

$$
\begin{aligned}
& A^{k} x \geq b^{k}-M^{k}\left(1-y_{k}\right), k \in K \\
& \sum_{k \in K} y_{k}=1, \quad y_{k} \in\{0,1\}, \quad k \in K
\end{aligned}
$$

where $M_{k}$ is set to a value sufficiently large that the $k$ th disjunct is not constraining when $y_{k}=0$. Thus the $k$ th disjunct is enforced when $y_{k}=1$, but through a different mechanism than in the convex hull formulation.

The formulation (30) is sharp when the $M^{k} \mathrm{~s}$ are as small as possible. This is achieved by observing that if $x$ does not belong to the polyhedron defined by the $k$ th disjunct, then it must belong to at least one of the other polyhedra. Thus allows us to set

$$
M^{k}=b^{k}-\min _{\ell \neq k}\left\{\min _{x}\left\{A^{k} x \mid A^{\ell} x \geq b^{\ell}\right\}\right\}
$$

where the minima are taken componenwise; that is, $\min \left\{\left(\alpha_{1}, \alpha_{2}\right),\left(\beta_{1}, \beta_{2}\right)\right\}=$ $\left(\min \left\{\alpha_{1}, \alpha_{2}\right\}, \min \left\{\beta_{1}, \beta_{2}\right\}\right)$. Computation of the big- $M \mathrm{~s}$ in this manner requires solution of $(|K|-1) \sum_{k \in K} m_{k}$ small linear programming problems $\min _{x}\left\{A_{i}^{k} x \mid A^{\ell} x \geq b^{\ell}\right\}$, where $m_{k}$ is the number of rows $A_{i}^{k}$ of $A^{k}$, but the resulting formulation contains no disaggregated variables $x^{k}$. The linear programming problems must obviously be bounded, but this is assured by the condition that the polyhedra have the same recession cone.

If finite bounds $L \leq x \leq U$ are available for the variables $x=\left(x_{1}, \ldots, x_{n}\right)$, big- $M$ s can be calculated more rapidly using the formula

$$
M^{k}=b^{k}-\sum_{j=1}^{n} \min \left\{0, A_{j}^{k}\right\} U_{j}-\sum_{j=1}^{n} \max \left\{0, A_{j}^{k}\right\} L_{j}
$$


where $A_{j}^{k}$ is column $j$ of $A^{k}$. The resulting big- $M \mathrm{~s}$, however, are in general larger than obtained by (31).

Sharp big- $M$ formulations are sometimes convex hull formulations. This is true, for example, of the sharp big- $M$ formulation for the fixed charge problem of Section 2.2. It simplifies to a formulation that is identical to the convex hull formulation (6). In other cases, however, a sharp big- $M$ formulation can provide a relaxation much weaker than the convex hull. For example, the disjunction

$$
\left(\begin{array}{l}
-x_{1}+x_{2} \geq 1 \\
x_{1}, x_{2} \in[0,2]
\end{array}\right) \vee\left(\begin{array}{c}
2 x_{1}-x_{2} \geq 2 \\
x_{1}, x_{2} \in[0,2]
\end{array}\right)
$$

has the sharp big- $M$ formulation

$$
\begin{aligned}
& -x_{1}+2 x_{2} \geq-1+2 y \\
& 2 x_{1}-x_{2} \geq 2-4 y \\
& x_{1}, x_{2} \in[0,2] \\
& y_{1}+y_{2}=1, y_{1}, y_{2} \in\{0,1\}
\end{aligned}
$$

The projection of the continuous relaxation onto $\left(x_{1}, x_{2}\right)$ is described by $x_{1}+x_{2} \geq 0, x_{1}, x_{2} \in[0,2]$ (Fig. 3). This is much weaker than the convex hull, which is described by $x_{1}+x_{1} \geq 1, x_{1}, x_{2} \in[0,2]$. In fact, it adds nothing to the box constraints $x_{1}, x_{2} \in[0,2]$ that are already part of both disjuncts.

Disjunctions of single linear inequalities (i.e., eack $m_{k}=1$ ) have special structure that allow one to eliminate the $0-1$ variables $y_{k}$ from a sharp big- $M$ formulation and obtain a relatively simple formulation [2]. This and other formulations are discussed in [3].

\section{Knapsack Modeling}

Mixed integer formulations frequently involve counting ideas that can be expressed as knapsack inequalities. For present purposes we can define a knapsack inequality to be any inequality of the form $a x \leq \alpha$, where some of the variables $x_{j}$ may be required to take nonnegative integer values. Variable $x_{j}$ can be interpreted as the quantity (integer or continuous) of item $j$ that is chosen for some purpose (perhaps to be placed in a knapsack). The left-hand side of the inequality therefore counts the total quantity selected, perhaps weighting some items differently than others. The right-hand side places a bound on the total weight (perhaps the knapsack capacity). 


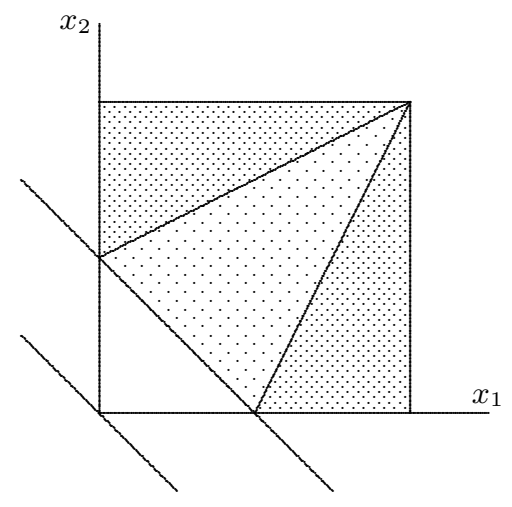

Figure 3: Feasible set of disjunction (32) (dark shaded area), convex hull of the feasible set (entire shaded area), and feasible set of the continuous relaxation of the sharp big- $M$ formulation (33) (entire box).

Problems of this sort include set packing, set covering, and set partitioning problems. Capital budgeting problems provide textbook examples. Countless other problems use constraints of this form, containing both continuous and integer-valued variables.

Knapsack constraints capture a very different modeling idea that the disjunctive constraints discussed earlier. The bounded mixed integer representability theorem (Theorem 1) technically accounts for knapsack problems, provided the variables are bounded, but only by brute force. For example, a single knapsack constraint with bounded integer variables defines a feasible set consisting of integer lattice points. The points can be regarded as finitely many recession cones that have the same recession cone (namely, the origin).

However, Theorem 1 can be generalized to account for knapsack constraints in a more natural way. This also enhances representability, because the integer variables need not be bounded. We begin with this task and then illustrate how problem formulation based on this result can lead to tight formulations.

\subsection{General Mixed Integer Representability}

It is convenient at this point to assume that mixed integer formulations consist of rational data. This has no practical repercussions but allows us to generalize the idea of a recession cone more easily. We also regard a polyhedron as a set of the form $\left\{x \in \mathbb{R}^{n} \mid A x \geq b\right\}$, where $A, b$ consist of 
rational data.

We define a subset $S$ of $\mathbb{R}^{n} \times \mathbb{Z}^{p}$ to be mixed integer representable if there is a constraint set of the following form whose projection onto $x$ is $S$ :

$$
\begin{aligned}
& A x+B u+D y \geq b \\
& x \in \mathbb{R}^{n} \times \mathbb{Z}^{p}, \quad u \in \mathbb{R}^{m}, y_{k} \in\{0,1\}, \text { all } k
\end{aligned}
$$

Let us say that a mixed integer polyhedron in $\mathbb{R}^{n+p}$ is the nonempty intersection of any polyhedron in $\mathbb{R}^{n+p}$ with $\mathbb{R}^{n} \times \mathbb{Z}^{p}$. We will show that a subset of $\mathbb{R}^{n} \times \mathbb{Z}^{p}$ is mixed integer representable if and only if it is the union of finitely many mixed integer polyhedra that have the same recession cone.

This requires that we define the recession cone of a mixed integer polyhedron. Let us say that rational vector $d$ is a recession direction of mixed integer polyhedron $P \subset \mathbb{R}^{n} \times \mathbb{Z}^{p}$ if it is a recession direction of some polyhedron $Q \subset \mathbb{R}^{n+p}$ for which $P=Q \cap\left(\mathbb{R}^{n} \times \mathbb{Z}^{p}\right)$. Then the recession cone of $P$ is the set of its recession directions. The definition is well formed because of the following lemma.

Lemma 3 All polyhedra in $\mathbb{R}^{n+p}$ having the same nonempty intersection with $\mathbb{R}^{n} \times \mathbb{Z}^{p}$ have the same recession cone.

Proof. Let $Q=\left\{x \in \mathbb{R}^{n+p} \mid A x \geq b\right\}$ and $Q^{\prime}=\left\{x \in \mathbb{R}^{n+p} \mid A^{\prime} x \geq b^{\prime}\right\}$ be polyhedra, and suppose that $Q \cap\left(\mathbb{R}^{n} \times \mathbb{Z}^{p}\right)=Q^{\prime} \cap\left(\mathbb{R}^{n} \times \mathbb{Z}^{p}\right)=P$, where $P$ is nonempty. It suffices to show that any recession direction $d$ of $Q$ is a recession direction of $Q^{\prime}$. Take any $u \in P$. Since $u \in Q$, we have $u+\alpha d \in Q$ for any $\alpha \geq 0$. Furthermore, because $d$ is rational, $u+\bar{\alpha} d \in Q \cap\left(\mathbb{R}^{n} \times \mathbb{Z}^{p}\right)$ for some sufficiently large $\bar{\alpha}>0$. Now if $d$ is not a recession direction of $Q^{\prime}$, then because $u \in Q^{\prime}$, we have $u+\beta \bar{\alpha} d \notin Q^{\prime}$ for some sufficiently large integer $\beta \geq 1$. Thus in particular $u+\beta \bar{\alpha} d \notin Q^{\prime} \cap\left(\mathbb{R}^{n} \times \mathbb{Z}^{p}\right)$. But because $\beta$ is integer, $u+\beta \bar{\alpha} d \in Q \cap\left(\mathbb{R}^{n} \times \mathbb{Z}^{p}\right)$. This violates the assumption that $Q, Q^{\prime}$ have the same intersection with $\mathbb{R}^{n} \times \mathbb{Z}^{p}$.

We can now state a necessary and sufficient condition for mixed integer representability. The proof is a straightforward extension of Jeroslow's proof $[4]$.

Theorem $4 A$ nonempty set $S \subset \mathbb{R}^{n} \times \mathbb{Z}^{p}$ is mixed integer representable if and only if $S$ is the union of finitely many mixed integer polyhedra in $\mathbb{R}^{n} \times \mathbb{Z}^{p}$ having the same recession cone. In particular, $S$ is mixed integer representable if and only if $S$ is the projection onto $x$ of a mixed integer 
formulation of the following form:

$$
\begin{aligned}
& x=\sum_{k \in K} x^{k} \\
& A^{k} x^{k} \geq b^{k} y_{k}, \quad k \in K \\
& \sum_{k \in K} y_{k}=1, \quad y_{k} \in\{0,1\}, k \in K \\
& x \in \mathbb{R}^{n} \times \mathbb{Z}^{p}
\end{aligned}
$$

Proof. Suppose first that $S$ is the union of mixed integer polyhedra $P_{k}, k \in K$, that have the same recession cone. Each $P_{k}$ has the form $\left\{x \mid A^{k} x^{k} \geq b^{k}\right\} \cap\left(\mathbb{R}^{n} \times \mathbb{Z}^{p}\right)$. It can be shown as follows that $S$ is represented by (35), and is therefore representable, because (35) has the form (34). Suppose first that $x \in S$. Then $x$ belongs to some $P_{k^{*}}$, which means that $x$ is feasible in (35) when $y_{k^{*}}=1, y_{k}=0$ for $k \neq k^{*}, x^{k^{*}}=x$, and $x^{k}=0$ for $k \neq k^{*}$. The constraint $A^{k} x^{k} \geq b^{k} y_{k}$ is satisfied by definition when $k=k^{*}$, and it is satisfied for other $k$ 's because $x^{k}=y_{k}=0$.

Now suppose that $x, y$ and $x^{k}$ satisfy (35). Let $Q_{k}=\left\{x \mid A^{k} x \geq b^{k}\right\}$, so that $P_{k}=Q_{k} \cap\left(\mathbb{R}^{n} \times \mathbb{Z}^{p}\right)$. To show that $x \in S$, note that exactly one $y_{k}$, say $y_{k^{*}}$, is equal to 1 . Then $A^{k^{*}} x^{k^{*}} \geq b^{k^{*}}$ is enforced, which means that $x^{k^{*}} \in Q_{k^{*}}$. For other $k^{\prime}$ s, $A^{k} x^{k} \geq 0$. Thus, $A^{k}\left(\beta x^{k}\right) \geq 0$ for all $\beta \geq 0$, which implies that $x^{k}$ is a recession direction for $Q_{k}$. Because by hypothesis all the $P_{k}$ s have the same recession cone, all $Q_{k} \mathrm{~s}$ have the same recession cone. Thus each $x^{k}\left(k \neq k^{*}\right)$ is a recession direction for $Q_{k^{*}}$, which means that $x=x^{k^{*}}+\sum_{k \neq k^{*}} x^{k}$ belongs to $Q_{k^{*}}$ and therefore to $\bigcup_{k \in K} Q_{k}$. But because $x \in \mathbb{R}^{n} \times \mathbb{Z}^{p}$, we have

$$
x \in\left(\bigcup_{k \in K} Q_{k}\right) \cap\left(\mathbb{R}^{n} \times \mathbb{Z}^{p}\right)=\bigcup_{k \in K}\left(Q_{k} \cap\left(\mathbb{R}^{n} \times \mathbb{Z}^{p}\right)\right)=\bigcup_{k \in K} P_{k}
$$

To prove the converse of the theorem, suppose that $S$ is represented by (34). To show that $S$ is a finite union of mixed integer polyhedra, let $P(\bar{y})$ be the set of all $x$ that are feasible in (34) when $y=\bar{y} \in\{0,1\}^{|K|}$. Because $S$ is nonempty, $P(\bar{y})$ is nonempty for at least one $\bar{y}$. Thus we let $Y$ be the set of all $\bar{y}$ for which $P(\bar{y})$ is nonempty. So $P(\bar{y})$ is a mixed integer polyhedron for all $\bar{y} \in Y$, and $S=\bigcup_{\bar{y} \in Y} P(\bar{y})$. To show that the $P(\bar{y})$ 's have the same recession cone, note that

$$
P(\bar{y})=\left\{x \in \mathbb{R}^{n} \times \mathbb{Z}^{p} \mid\left[\begin{array}{rrr}
A & B & D \\
0 & 0 & 1 \\
0 & 0 & -1
\end{array}\right]\left[\begin{array}{l}
x \\
u \\
y
\end{array}\right] \geq\left[\begin{array}{r}
b \\
\bar{y} \\
-\bar{y}
\end{array}\right] \text { for some } u, y\right\}
$$


But $x^{\prime}$ is a recession direction of $P(\bar{y})$ if and only if $\left(x^{\prime}, u^{\prime}, y^{\prime}\right)$ is a recession direction of

$$
\left\{\left[\begin{array}{l}
x \\
u \\
y
\end{array}\right] \in \mathbb{R}^{n} \times \mathbb{Z}^{p} \times \mathbb{R}^{m+|K|} \mid\left[\begin{array}{rrr}
A & B & D \\
0 & 0 & 1 \\
0 & 0 & -1
\end{array}\right]\left[\begin{array}{l}
x \\
u \\
y
\end{array}\right] \geq\left[\begin{array}{r}
b \\
\bar{y} \\
-\bar{y}
\end{array}\right]\right\}
$$

for some $u^{\prime}, y^{\prime}$. The latter is true if and only if

$$
\left[\begin{array}{rrr}
A & B & D \\
0 & 0 & 1 \\
0 & 0 & -1
\end{array}\right]\left[\begin{array}{l}
x^{\prime} \\
u^{\prime} \\
y^{\prime}
\end{array}\right] \geq\left[\begin{array}{l}
0 \\
0 \\
0
\end{array}\right]
$$

This means that the recession directions of $P(\bar{y})$ are the same for all $\bar{y} \in Y$, as desired.

The theorem says in part that any nonempty mixed integer representable subset of $\mathbb{R}^{n} \times \mathbb{Z}^{p}$ is the feasible set of some disjunction

$$
\bigvee_{k \in K}\left(\begin{array}{c}
A^{k} x \geq b^{k} \\
x \in \mathbb{R}^{n} \times \mathbb{Z}^{p}
\end{array}\right)
$$

This and the following lemma give us a technique for writing a convex hull formulation by conceiving the feasible set as a union of mixed integer polyhedra.

Lemma 5 If each disjunct of (36) is a convex hull formulation, then (35) is a convex hull formulation of (36).

Proof. It is clear that $x$ satisfies (36) if any only if $x$ satisfies (35) for some $\left(x^{k}, y_{k} \mid k \in K\right)$. It remains to show that, given any feasible solution $\bar{x},\left(\bar{x}^{k}, \bar{y}_{k} \mid k \in K\right)$ of the continuous relaxation of (35), $\bar{x}$ belongs to the convex hull of the feasible set of (36). But $\bar{x}$ is the convex combination

$$
\bar{x}=\sum_{k \in K^{+}} \bar{y}_{k} \frac{\bar{x}^{k}}{\bar{y}_{k}}
$$

where $K^{+}=\left\{k \in K \mid \bar{y}_{k}>0\right\}$. Furthermore, each point $\bar{x}^{k} / \bar{y}_{k}$ satisfies $A^{k}\left(\bar{x}^{k} / \bar{y}_{k}\right) \geq b^{k}$ because $\left(\bar{x}^{k}, \bar{y}_{k}\right)$ satisfies $A^{k} \bar{x}^{k} \geq b^{k} \bar{y}_{k}$. Thus $\bar{x}^{k} / \bar{y}_{k}$ satisfies the continuous relaxation of the $k$ th disjunct of (36) and so, by hypothesis, belongs to the convex hull of the feasible set of that disjunct. This and (37) imply that $\bar{x}$ belongs to the convex hull of the feasible set of (36). 


\subsection{Big- $M$ Mixed Integer Disjunctive Formulations}

As noted earlier, any set that is bounded mixed integer representable can be represented by a big- $M$ as well as a convex hull disjunctive formulation [8]. This is likewise true of general mixed integer representable sets. Let us say that a sharp big-M mixed integer disjunctive formulation has the form

$$
\begin{aligned}
& A^{k} x \geq b^{k}-M^{k}\left(1-y_{k}\right), \quad k \in K \\
& x \in \mathbb{R}^{n} \times \mathbb{Z}^{p}, \quad \sum_{k \in K} y_{k}=1, \quad y_{k} \in\{0,1\}, \quad k \in K
\end{aligned}
$$

where

$$
M^{k}=b^{k}-\min _{\ell \neq k}\left\{\min _{x}\left\{A^{k} x \mid A^{\ell} x \geq b^{\ell}, x \in \mathbb{R}^{n} \times \mathbb{Z}^{p}\right\}\right\}
$$

Theorem 6 If set $S \subset \mathbb{R}^{n} \times \mathbb{Z}^{p}$ is the union of finitely many mixed integer polyhedra $P_{k}=Q_{k} \cap\left(\mathbb{R}^{n} \times \mathbb{Z}^{p}\right)$ (for $k \in K$ ) having the same recession cone, where $Q_{k}=\left\{x \mid A^{k} x \geq b^{k}\right\}$, then $S$ is represented by the sharp big-M mixed integer disjunctive formulation (38).

Proof. System (38) clearly represents $S$ if every component of $M^{k}$ as given by (39) is finite. We therefore suppose that some component $i$ of some $M^{k}$ is infinite, which implies that $\min \left\{A_{i}^{k} x \mid A^{\ell} x \geq b^{\ell}\right\}$ is unbounded for some $\ell \neq k$. Since $P_{\ell}$ is nonempty, this means there is a point $\bar{x} \in P_{\ell}$ and a rational direction $d$ such that $A_{i}^{k}(\bar{x}+\alpha d)$ is unbounded in a negative direction as $\alpha \rightarrow \infty$, and such that $\bar{x}+\alpha d \in Q_{\ell}$ for all $\alpha \geq 0$. This means $d$ is a recession direction of $P_{\ell}$ and therefore, by hypothesis, a recession direction of $P_{k}$. Thus by Lemma $3, d$ is a recession direction of $Q_{k}$. Since $P_{k}$ is nonempty, there is an $x^{\prime}$ satisfying $A^{k} x^{\prime} \geq b^{k}$, and for any such $x^{\prime}$ we have $A^{k}\left(x^{\prime}+\alpha d\right) \geq b^{k}$ for all $\alpha \geq 0$. Thus $A^{k}(\bar{x}+\alpha d) \geq b^{k}+A^{k}\left(\bar{x}-x^{\prime}\right)$ for all $\alpha \geq 0$, which means that $A_{i}^{k}(\bar{x}+\alpha d)$ cannot be unbounded in a negative direction as $\alpha \rightarrow \infty$.

\subsection{Example: Facility Location}

An extension of the capacitated facility location problem considered earlier illustrates the usefulness of extending representability to disjunctions of mixed integer systems. Before, the cost of transporting quantity $x_{i j}$ from facility location $i$ to customer $j$ was a continuous quantity $c_{i j} x_{i j}$. Now we suppose that goods transported on route $(i, j)$ must be loaded into one or 
more vehicles, each with capacity $K_{i j}$, where each vehicle incurs a fixed cost $c_{i j}$. If $w_{i j}$ is the number of vehicles used, then we have a disjunction of mixed integer systems for each location $i$ :

$$
\left(\begin{array}{c}
\sum_{j=1}^{n} x_{i j} \leq C_{i} \\
0 \leq x_{i j} \leq K_{i j} w_{i j}, \text { all } j \\
z_{i}=f_{i} \\
w_{i j} \in \mathbb{Z} \text {, all } j
\end{array}\right) \vee\left(\begin{array}{c}
x_{i j}=0, \text { all } j \\
z_{i}=0
\end{array}\right)
$$

The mixed integer polyhedra defined by the two disjuncts have different recession cones. The cone for the first polyhedron is $\left\{\left(x_{i}, w_{i}\right) \mid x_{i}=0, w_{i} \geq 0\right\}$ where $x_{i}=\left(x_{i 1}, \ldots, x_{i n}\right)$ and $w_{i}=\left(w_{i 1}, \ldots, w_{i n}\right)$, while the cone for the second is $\left\{\left(x_{i}, w_{i}\right) \mid x_{i}=0\right\}$. However, if we add the innocuous constraint $w_{i} \geq 0$ to the second disjunct, the two disjuncts have the same recession cone and can therefore be given a convex hull formulation:

$$
\begin{aligned}
& \sum_{j=1}^{n} x_{i j} \leq C_{i} y_{i}, \text { all } i \\
& 0 \leq x_{i j} \leq K_{i j} w_{i j}, \text { all } j \\
& z_{i}=f_{i} y_{i}, \quad y_{i} \in\{0,1\}, \quad w_{i j} \in \mathbb{Z}, \text { all } j
\end{aligned}
$$

This yields a mixed integer formulation for the problem:

$$
\begin{aligned}
& \min \sum_{i=1}^{m}\left(f_{i} y_{i}+\sum_{j=1}^{n} c_{i j} w_{i j}\right) \\
& \sum_{j=1}^{n} x_{i j} \leq C_{i} y_{i}, \text { all } i \\
& 0 \leq x_{i j} \leq K_{i j} w_{i j}, \text { all } i, j \\
& \sum_{i=1}^{m} x_{i j}=D_{j}, \text { all } j \\
& y_{i} \in\{0,1\}, w_{i j} \in \mathbb{Z}, \text { all } i, j
\end{aligned}
$$

Using a sharp big- $M$ mixed integer formulation in place of the convex hull formulation (41) yields the same problem formulation (42).

\subsection{Example: Package Delivery}

A final example, adapted from [1,6], illustrates how the approach presented here can result in a formulation that is superior to the standard formulation. 
A collection of packages are to be delivered by several trucks, and each package $j$ has size $a_{j}$. Each available truck $i$ has capacity $Q_{i}$ and $\operatorname{costs} c_{i}$ to operate. The problem is to decide which trucks to use, and which packages to load on each truck, to deliver all the items at minimum cost.

We will formulate the problem by analyzing it as a combination of knapsack and disjunctive ideas. The decision problem consists of two levels: the choice of which trucks to use, followed by the choice of which packages to load on each truck. The trucks selected must provide sufficient capacity, which leads naturally to a 0-1 knapsack constraint:

$$
\sum_{i=1}^{m} Q_{i} y_{i} \geq \sum_{j=1}^{n} a_{j}
$$

where each $y_{i} \in\{0,1\}$ and $y_{i}=1$ when truck $i$ is selected.

The secondary choice of which packages to load on truck $i$ depends on whether that truck is selected. This suggests a disjunction of two alternatives. If the truck $i$ is selected, then a cost $c_{i}$ is incurred, and the items loaded must fit into the truck (a 0-1 knapsack constraint). If truck $i$ is not selected, then no items can be loaded (another knapsack constraint). The disjunction is

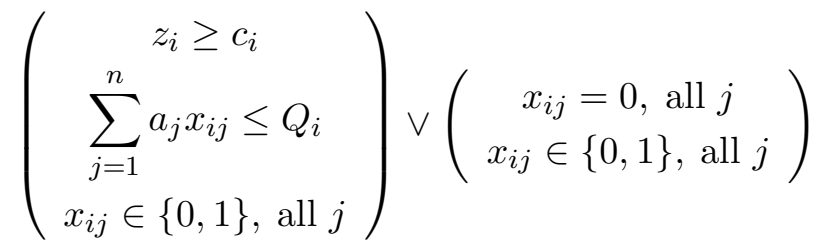

where $z_{i}$ is the fixed cost incurred by truck $i$, and $x_{i j}=1$ when package $j$ is loaded into truck $i$. The feasible set is the union of two mixed integer polyhedra. They have the same recession cone if we add $z_{i} \geq 0$ to the second disjunct. If we suppose $y_{i}=1$ when the first disjunct is enforced, the convex hull formulation of (44) is

$$
\begin{aligned}
& z_{i} \geq c_{i} y_{i} \\
& \sum_{j=1}^{n} a_{j} x_{i j} \leq Q_{i} y_{i} \\
& y_{i}, x_{i j} \in\{0,1\}, \text { all } j
\end{aligned}
$$

Finally, we make sure that each packaged must be shipped, which poses a set of knapsack constraints:

$$
\sum_{i=1}^{m} x_{i j} \geq 1, \quad x_{i j} \in\{0,1\}, \text { all } j
$$


Since (43) and (46) can be viewed as disjunctions having one disjunct, we have conceived the problem as consisting of disjunctions of mixed integer systems. If we minimize total fixed cost $\sum_{i} z_{i}$ subject to (43), (45), and (46), the resulting mixed integer model immediately simplifies to

$$
\begin{aligned}
& \min \sum_{i=1}^{m} c_{i} y_{i} \\
& \sum_{i=1}^{m} Q_{i} y_{i} \geq \sum_{j=1}^{n} a_{j} \\
& \sum_{j=1}^{n} a_{j} x_{i j} \leq Q_{i} y_{i}, \text { all } i \\
& \sum_{i=1}^{m} x_{i j} \geq 1, \quad x_{i j} \in\{0,1\}, \text { all } j \\
& y_{i} \in\{0,1\}, \quad x_{i j} \in\{0,1\}, \text { all } i, j
\end{aligned}
$$

This formulation differs in two ways from a formulation that one might initially write for this problem. First, one might omit the factor $y_{i}$ from constraints (c), because these constraints ensure that each truck's load is within that truck's capacity. It is therefore natural to write simply the capacity $Q_{i}$ on the right-hand side. However, a fairly well-known modeling "trick" is to write $Q_{i} y_{i}$ instead, because this retains the validity of the formulation while making its continuous relaxation tighter. The approach recommended here allows one to derive the tighter formulation without knowing the "trick" in advance.

Second, a standard formulation would not contain constraint (b), because due to (d) it is implied by the sum of constraints (c). According to conventional wisdom, there is no point is writing a constraint that is a nonnegative linear combination of other constraints. However, it is reported in [6] that the problem is far easier to solve with constraint (b) than without it, because the presence of (b) allows the solver to deduce lifted knapsack cuts, which create a much tighter continuous relaxation. Thus in this instance, a principled approach enables one to write a formulation that is superior to the standard one.

\section{Conclusion}

We have suggested how mixed integer problem formulation can be undertaken in a principled way. We by no means provide a method by which one 
can mechanically generate mixed integer formulations. Problem formulation remains an irreducibly creative act. Yet the framework presented here can give some guidance as to how to proceed.

Problems often pose choices between alternatives, and these can be represented as disjunctions of inequality systems. Counting ideas can be represented as integer knapsack constraints that appear among the inequality constraints. The disjunctions can be given convex hull or big- $M$ formulations, resulting in a mixed integer formulation for the problem.

There may be a good deal of latitude as to how to view a problem as containing disjunctive and counting elements. Different interpretations of the problem can lead to different formulations. Even when the disjunctive constraints have been written, there is the issue as to whether some of them should be combined to obtain a tighter formulation.

Once the disjunctive constraints are finalized, the mixed integer formulation of each disjunct typically allows simplification. It may be possible to automate the simplification process, and this presents an interesting issue for future research.

Several additional research issues remain. (a) Are there sufficient conditions under which a big- $M$ disjunctive formulation is a convex hull formation? (b) When is it advantageous to use a big- $M$ rather than a convex hull disjunctive formulation? (c) Are there sufficient conditions under which a formulation containing logical constraints is a convex hull formulation? (d) When is it advantageous to replace logical constraints with convex hull disjunctive formulations?

In general, mixed integer problem formulation deserves more serious study that it has received. Jeroslow's work was a significant contribution, but much remains to be done. If the formulation process is better understood, it may be possible to develop more effective tools to assist practitioners in formulating problems. This in turn will allow more applications to benefit from the powerful solution technology that has been developed for mixed integer programming.

\section{References}

[1] K. Aardal. Reformulation of capacitated facility location problems: How redundant information can help. Annals of Operations Research, 82:289309, 1998.

[2] N. Beaumont. An algorithm for disjunctive programs. European Journal of Operational Research, 48:362-371, 1990. 
[3] J. N. Hooker. Integrated Methods for Optimization. Springer, New York, 2007.

[4] R. G. Jeroslow. Representability in mixed integer programming, I: Characterization results. Discrete Applied Mathematics, 17:223-243, 1987.

[5] R. G. Jeroslow. Logic-Based Decision Support: Mixed Integer Model Formulation. Annals of Discrete Mathematics. North-Holland, 1989.

[6] M. Trick. Formulations and reformulations in integer programming. In R. Barták and M. Milano, editors, Integration of AI and OR Techniques in Constraint Programming for Combinatorial Optimization Problems (CPAIOR 2005), volume 3524 of Lecture Notes in Computer Science, pages 366-379. Springer, 2005.

[7] H. P. Williams. Model Building in Mathematical Programming, 4th Ed. Wiley, New York, 1999.

[8] H. P. Williams. Logic and Integer Programming. Springer, to appear. 